\title{
Forum
}

\section{Quelques remarques sur les noyaux durs des sciences molles}

\section{Un exemple pris dans l'anthropologie}

\author{
Maurice Godelier \\ Anthropologue, directeur d'études, EHESS, 54 boulevard Raspail, 75006 Paris, France
}

Ce titre est volontairement ironique et provocateur. Pour beaucoup de scientifiques des sciences dites «dures", les sciences sociales sont à peine des sciences. Ce sont au mieux des discours littéraires qui expriment sous forme de pseudo-raisonnements les positions idéologiques de leurs auteurs. Là encore je caricature, mais je ne m'indigne pas personnellement lorsque l'on dénonce comme "pseudo-scientifiques » des textes qui mélangent de façon éclectique des idées de Derrida, Foucault, Lyotard et d'autres auteurs élevés, sans qu'ils l'aient toujours vraiment voulu, à la dignité de gourous de la pensée.

Il est indéniable que souvent, dans les textes de certains auteurs, les faits manquent pour appuyer les idées, et les conclusions sont affirmées sans que des prémisses vérifiables aient été proposées. Aujourd'hui, je voudrais montrer qu'il existe des approches, des méthodes au sein des sciences sociales qui permettent d'éclairer les logiques de fonctionnement des sociétés, ainsi que les modes de pensée des acteurs engagés dans la reproduction et l'évolution de ces sociétés. Bref, je voudrais montrer que l'histoire humaine, dans la diversité de ses systèmes sociaux et culturels, n'échappe pas à la pensée «scientifique ». Celle-ci n'a donc pas seulement le privilège de percer les modes d'organisation de la matière animée ou inanimée, de la nature qui entoure l'homme et qui en précède l'existence.

L'objet des sciences sociales est de reconstruire, si l'on se tourne vers le passé, ou de découvrir, si l'on se tourne vers le présent, la logique des rapports sociaux et des représentations qui caractérisent telle ou telle forme de société, à telle ou telle époque. Reconstruire des logiques

Auteur correspondant : Maurice.Godelier@ehess.fr sociales et historiques ne signifie pas prendre pour explication dernière les représentations que les acteurs euxmêmes se faisaient, ou se font, de ces logiques, de leur place dans leur société. Mais il ne peut pas y avoir d'analyse des formes d'existence sociale développées au cours de l'histoire de l'humanité, qui ne prenne en compte les représentations que les individus et les groupes vivant dans une société se font de celle-ci et de leur propre place en son sein.

Venons-en à une discipline que je pratique et connais de l'intérieur, l'anthropologie sociale. Cette discipline est née de l'expansion même de l'Occident à partir du $\mathrm{XVI}^{\mathrm{e}}$ siècle, expansion commerciale, militaire, politique, idéologique. Dans ce processus, des centaines de sociétés organisées différemment ont été découvertes par les Occidentaux et, pour beaucoup d'entre elles, conquises et subordonnées. Partout, pour administrer ces sociétés, dans un cadre colonial, pour convertir au christianisme des peuples aux religions très différentes (parfois égales en force avec le christianisme comme l'islam ou l'hindouisme, mais souvent moins puissantes parce que sans ambition de conversion comme les religions tribales), administrateurs, militaires, missionnaires, voyageurs, commerçants ont accumulé des observations et des informations jugées par eux utiles pour atteindre leurs buts. Bien entendu ces observations étaient nécessairement biaisées par ces buts (évangéliser, pacifier, etc.).

Ce fut là le premier matériau de base de l'anthropologie. La rupture avec cette ethnologie spontanée et intéressée s'est faite avec l'œuvre de Henri Lewis Morgan qui, au milieu du XIX ${ }^{\mathrm{e}}$ siècle, avait fait des recherches sur le terrain parmi les tribus indiennes d'Amérique du Nord. Cette rupture a commencé lorsqu'il a constaté que les Indiens de la confédération iroquoise traçaient 
(1)

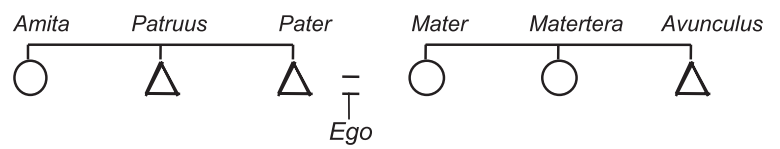

(2)

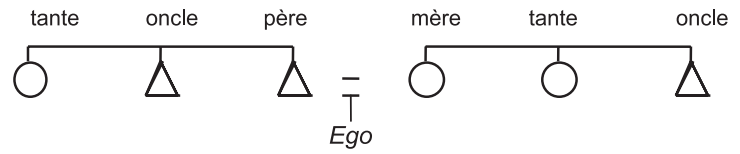

Fig. 1. (1) terminologie latine et (2) terminologie française.

la descendance des individus par les femmes, et que les enfants d'un mariage appartenaient non pas au clan du mari, mais à celui de la mère et du frère de la mère. Il a constaté également, pour l'épouse, qu'au lieu d'aller vivre dans le clan de son époux, c'était celui-ci qui venait résider dans son clan. Bref, Morgan en a conclu que les rapports de parenté dans cette société possédaient une logique propre mais différente de celle des sociétés occidentales. De là l'idée de continuer à recueillir des informations sur d'autres tribus d'Amérique du Nord (82 d'entre elles), et ensuite de lancer une enquête mondiale auprès de 500 correspondants, répartis dans les pays colonisés par l'Occident, ou en contact avec lui.

Et c'est là que commença à se construire le premier noyau dur de l'anthropologie, c'est-à-dire l'un des noyaux durs des sciences sociales. Il y en a d'autres dans les autres disciplines. Les résultats de l'enquête mondiale de Morgan, qui fut suivie pendant des générations par des dizaines d'enquêtes complémentaires réalisées par des anthropologues, devenus cette fois des professionnels, ont montré que les milliers de sociétés coexistant de par le monde ont toutes des systèmes et des terminologies de parenté différents, mais que cette diversité peut se ramener à des variantes de sept grands types de terminologies de parenté : les types dravidien, australien, iroquois, crow-omaha, hawaïen, soudanais et eskimo. Le système français actuel est un système de type "eskimo » et il dérive par transformation du système latin antique, qui était un système dit de type "soudanais".

Pour faire bref, chacun sait qu'en latin, dans l'Antiquité, on distinguait par des termes différents (Fig. 1, ligne 1) : le père (pater), le frère du père (patruus), la sœur du père (amita), la mère (mater), la sœur de la mère (matertera) et le frère de la mère (avunculus). Ce qui veut dire que les collatéraux étaient distingués des parents en ligne directe, et des termes distincts désignaient les différents types de cousins par rapport à Ego. C'est ce qu'on appelle une terminologie de type «bifurcate collateral ${ }^{1}$ » et que $1^{\prime}$ on trouve encore aujourd'hui, par exemple, au sein des populations du Soudan, d'où l'appellation «terminologie

\footnotetext{
${ }^{1}$ Le vocabulaire utilisé internationalement par les anthropologues pour décrire les systèmes de parenté est habituellement en anglais. J'ai préféré garder cette langue pour les expressions qui exigeraient de trop longues périphrases pour être traduites en français.
}

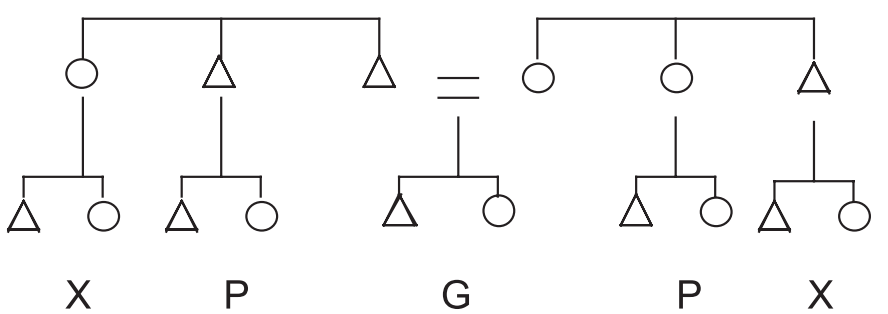

Fig. 2. Germains et cousins. $G=$ germains ; $P=$ cousins parallèles $\| ; X=$ cousins croisés $X$.

de type soudanais ». Ce que l'on appelle «bifurcation» est le fait que des parents du $2^{\mathrm{e}}$, du $3^{\mathrm{e}}$, voire du $n^{\mathrm{e}}$ degré, sont liés à Ego par un parent qui les connecte à lui, et qui est soit un homme, soit une femme. Dans les systèmes qui reconnaissent ce critère de classification, la conséquence est que l'on désigne ces individus apparentés à Ego par des termes différents selon que l'individu qui les connecte à lui est un homme ou une femme. Cela éclaire la distinction qui est faite dans de nombreux systèmes entre les cousins parallèles et les cousins croisés. Les cousins parallèles sont les enfants des frères du père ou des sœurs de la mère, donc d'ascendants du même sexe que du père, que de la mère. Les cousins croisés sont les enfants des ascendants du sexe opposé à celui du père et à celui de la mère, donc les enfants des sœurs du père ou des frères de la mère (Fig. 2).

La terminologie de parenté actuelle des Français, mais aussi des Anglais, etc., est de type "eskimo », ceci parce que sa structure formelle se retrouve également chez les Inuit (terme qui remplace aujourd'hui le terme péjoratif $\mathrm{d}^{\prime}$ Eskimo autrefois en usage). Nous avons un terme pour "père », un terme pour «mère », et les frères du père et de la mère sont désignés, eux, par un seul terme, c'est-à-dire «oncle», en anglais « uncle», en allemand «Onkel», etc. Les sœurs du père et de la mère sont désignées, elles, par un seul terme, " tante » (aunt, etc.). Or il est intéressant de savoir qu'en français, le terme " oncle » est une transformation d'avunculus et "tante» d'amita. Il s'est donc passé historiquement que le terme "patruus» a disparu et que le terme "avunculus", qui désignait au départ seulement l'oncle maternel, en est venu à désigner aussi l'oncle paternel. Symétriquement le terme "matertera " disparaissait et le terme "amita», qui à l'origine désignait seulement la sœur du père, a désigné également la sœur de la mère. Automatiquement ces transformations devaient en entraîner d'autres, ce qui veut dire que les terminologues désignent des rapports qui « font système ». Les enfants des oncles paternels et maternels ou des tantes paternelles et maternelles, qui en latin étaient désignés par des termes différents, sont tous devenus des «cousins » d'Ego. Nous voyons donc qu'au terme d'un processus historique qui s'est développé peut-être déjà à la fin de la république romaine, et dont on ne connaît pas encore les raisons, un système terminologique, le système 
Tableau 1. Les caractéristiques des quatre grandes catégories de terminologie.

\begin{tabular}{|c|c|c|c|}
\hline Types & Collatéralité & Bifurcation & Terminologies \\
\hline «Bifurcate collateral » & + & + & Soudanais \\
\hline \multirow{4}{*}{ «Bifurcate merging» } & \multirow{4}{*}{-} & \multirow{4}{*}{+} & Dravidien \\
\hline & & & Iroquois \\
\hline & & & Crow-Omaha \\
\hline & & & Australien \\
\hline «Lineal » & + & - & Eskimo \\
\hline «Generational » & - & - & Hawaïen \\
\hline
\end{tabular}

latin de type "soudanais " s'est transformé en système européen de type " eskimo ». Les systèmes eskimo sont dits des systèmes «linéaires » parce que les collatéraux sont distingués des parents en ligne directe, mais sont rassemblés sous le même terme quel que soit leur côté, paternel ou maternel, par rapport à Ego : exemple Figure 1 (ligne 2) pour les termes « oncle» et «tante».

Finalement, pour faire bref, toutes les terminologies connues aujourd'hui se regroupent dans quatre grandes catégories, selon que les collatéraux sont distincts ou non. On peut les représenter par le Tableau 1. Je simplifie, car on a trouvé des terminologies qui combinent des traits dravidiens et des traits iroquois, et qui apparaissent comme des formes de transition entre deux types, mais des formes de transition stabilisées, donc intermédiaires, entre les deux types.

On remarque tout de suite que ces terminologies forment une combinatoire. Il existe ainsi des systèmes où la bifurcation entre les lignes collatérales et directes est effacée («bifurcate merging»), tels les systèmes dits «iroquois ». Dans ce type de système, un même terme désigne le père et tous les frères du père, qui sont appelés "pères ». Un autre terme désigne la mère et toutes les sœurs de la mère, qui sont appelées «mères ». Donc Ego n'a pas d'oncle du côté de son père, il n'a que des " pères ». Il n'a pas de tante du côté de sa mère, il n'a que des «mères ». Par contre il existe un terme spécifique pour la sœur du père et un autre pour le(s) frère(s) de la mère.

Donc dans un système "iroquois », tous les enfants des frères de mon père, étant des enfants de "pères ", sont mes frères et sœurs. De même tous les enfants des sœurs de ma mère sont des enfants de "mères ", donc des frères et sœurs. La catégorie de frère et sœur, c'est-àdire la catégorie des germains s'étend ainsi à toute une série d'individus qui, dans un système européen, sont désignés comme des cousins.

Il est également important de voir comment les systèmes classent les germains et les cousins. Dans beaucoup de sociétés, les enfants d'une sœur de père, ou d'un frère de mère, sont désignés par des termes différents de ceux employés pour les enfants de frères de père ou de sœurs
Tableau 2. Différents types de distinctions entre germains, cousins parallèles et croisés.

\begin{tabular}{lc}
\hline Types & $\begin{array}{c}\text { Distinctions entre germains } \\
\text { et cousins parallèles et croisés }\end{array}$ \\
\hline Soudanais & $G \neq P \neq X_{\mathrm{p}} \neq X_{\mathrm{m}}$ \\
\hline $\begin{array}{l}\text { Dravidien } \\
\text { Australien } \\
\text { Iroquois }\end{array}$ & $(G=P) \neq X$ \\
\hline Crow-Omaha & $(G=P) \neq X_{\mathrm{p}}$ et de $X_{\mathrm{m}}$ \\
\hline Eskimo (européen) & $G \neq P=X$ \\
\hline Hawaïen & $G=P=X$ \\
\hline
\end{tabular}

de mère. Dans le vocabulaire anthropologique, nous venons de le voir, on désigne les premiers par les termes de "cousins croisés », parce qu'on passe, pour arriver à eux, par un ascendant du sexe opposé à celui du père (= sœur du père) ou de la mère (= le frère de la mère), et on appelle les enfants des frères du père ou des sœurs de la mère des « cousins parallèles ». Bref, à partir de ces trois catégories, les germains, frères et sœurs réels (ou classificatoires) d'Ego, les cousins croisés et les cousins parallèles, on a découvert que les différentes terminologies et systèmes de parenté opéraient des fusions ou des distinctions qui expriment leur logique profonde. Dans les systèmes européens, le mot « cousin » désigne aussi bien les enfants des tantes que des oncles des deux côtés, paternel et maternel. Donc dans ces systèmes, les cousins parallèles et les cousins croisés sont équivalents et la distinction entre eux n'existe pas. Tous les cousins se distinguent donc et s'opposent aux germains (mes frères et sœurs). La formule est $G \neq(P=X)$. $G=$ germains, $P=$ parallèles, $X=$ croisés. Si l'on prend le système latin ancien, les germains étaient désignés par un terme différent de celui utilisé pour les cousins parallèles, et ceux-ci étaient distingués des cousins croisés du côté du père, qui eux-mêmes étaient distingués des croisés du côté de la mère. La formule est: $G \neq P \neq X_{\mathrm{p}} \neq X_{\mathrm{m}}$. ( $X_{\mathrm{p}}=$ cousins croisés du côté du père, $X_{\mathrm{m}}$ du côté de la mère).

Bref, pour résumer en un tableau ces transformations, on aboutit aux formules suivantes (Tab. 2).

Dans les systèmes hawaïens, il n'y a pas de distinction entre germains et cousins. Tous sont des germains. Ceci est la conséquence du fait qu'à la génération $G+1$, il n'y a pas de distinction entre le «père » et les « oncles » du côté paternel et du côté maternel, ni entre la " mère » et les «tantes" du côté paternel et du côté maternel. On n'a donc que deux termes, "père » et «mère », appliqués à toute une série d'individus qui sont distingués dans d'autres systèmes. Leurs enfants sont donc, logiquement, entre eux comme des frères et des sœurs.

Allons plus loin. Tous les systèmes de parenté peuvent également être répartis entre deux catégories, selon que les affins (c'est-à-dire les personnes que l'on 
Tableau 3. Terminologies de parenté avec ou sans vocabulaire pour les affins.

\begin{tabular}{ll}
\hline Terminologies sans vocabulaire & Australien \\
d'affinité distinct & Dravidien \\
& Hawaïen \\
\hline Terminologies distinguant un vocabulaire & Iroquois \\
pour les consanguins et un autre pour & Crow-Omaha \\
les affins & Eskimo \\
& Soudanais \\
\hline
\end{tabular}

épouse, les alliés par le mariage) sont également des parents consanguins ou ne le sont pas. Pour donner un exemple, dans les systèmes dravidiens existe souvent une règle préférentielle de mariage selon laquelle un homme doit épouser la fille du frère de sa mère, de son oncle maternel. De ce fait, dans la langue il n'y a qu'un seul terme pour désigner l' « oncle maternel » et le «beau-père». Ceci implique que le mariage, dans ces sociétés, repose sur l'échange des femmes entre deux groupes, et que la sœur de mon père est en même temps l'épouse du frère de ma mère. Bref, dans certains systèmes de parenté, il n'y a donc pas de vocabulaire distinct pour désigner les alliés, puisque le même terme désigne un consanguin (oncle maternel) qui est en même temps un allié potentiel (beaupère). Dans les autres systèmes, il existe des vocabulaires spécifiques pour désigner les alliés - comme en français les mots «beau-père », «belle-mère », «belle-sœur », et en anglais «father-in-law», «mother-in-law», « sister-in-law», etc. (Tab. 3).

Pour terminer il nous faut examiner deux autres dimensions de la parenté, l'existence de principes de descendance et l'existence de différentes formes d'échanges organisant l'alliance.

Là encore, il n'existe que quatre principes de descendance, selon que l'on compte la descendance par les hommes exclusivement (systèmes patrilinéaires, exemple : Baruya de Nouvelle-Guinée), ou par les femmes exclusivement (systèmes matrilinéaires, exemple : les Iroquois d'Amérique du Nord). Ces deux principes engendrent des systèmes dits " unilinéaires ». Par contre, les systèmes européens ne font pas de grande différence entre la descendance par les hommes ou par les femmes, ce sont des systèmes «indifférenciés ». Il existe aussi des systèmes " ambi-bilinéaires » où l'individu reçoit une part de son statut en ligne paternelle et une autre en ligne maternelle. Nous passons rapidement sur quelques complications (existence de systèmes bilinéaires parallèles : les fils appartiennent au clan du père et les filles au clan de la mère, et systèmes bilinéaires croisés où les filles appartiennent au clan du père et les fils au clan de la mère) (Fig. 3). Dans le monde, les systèmes patrilinéaires sont dominants $(47 \%)$, les systèmes indifférenciés tels les systèmes européens sont en seconde position (à peu près $30 \%$ ), les systèmes matrilinéaires font $18 \%$ et les systèmes ambi- et bilinéaires comptent pour $5 \%$.

Dernier point, les règles de mariage dans la plupart des cas reposent sur des formes d'échange. Là encore trois possibilités ont été réalisées par l'humanité. Soit les hommes échangent entre eux des femmes (une grande partie des systèmes), soit les femmes échangent entre elles les hommes (exemple : les Rhadès du Vietnam), soit enfin hommes et femmes quittent leurs familles pour en créer d'autres (systèmes européens et euro-américains par exemple). Comme nous avons affaire à des échanges de «dons » de personnes, les positions des donneurs et des preneurs peuvent être soit différentes soit équivalentes. Ici encore trois possibilités ont été réalisées : 1) les donneurs sont supérieurs aux preneurs ; 2) les preneurs sont supérieurs aux donneurs ; 3) preneurs et donneurs sont de statuts équivalents. Mais il ne faudrait pas oublier que, dans certaines sociétés, des mariages frèressœurs étaient autorisés, correspondant à des alliances sans échange.

Je terminerai là cet exposé excessivement sommaire de quelques résultats acquis par plus d'un siècle de recherches anthropologiques. On voit donc que, comme les sciences de la nature, les sciences sociales aboutissent à réduire la complexité et la diversité du réel à un certain nombre de configurations de rapports sociaux construites historiquement à partir d'un petit nombre de principes. Cependant on ne sait pas encore quelles sont les raisons sociales et les conditions historiques qui ont amené l'apparition et le développement dans telle et telle société de telle et telle configuration, à telle ou telle époque. On ne connaît donc pas les raisons de la distribution inégale de ces systèmes sur la surface de la planète Terre.

Les anthropologues proposent cependant diverses hypothèses sur lesquelles je ne dirai rien ici. Je ferai seulement remarquer aux collègues des sciences «dures » que les sociétés ne sont pas des objets que l'on peut faire entrer dans un système de machines et sur lesquelles on pourrait se livrer à des "expériences ». Seule l'histoire est le lieu des expérimentations que les individus et les groupes tentent pour préserver, abandonner, détruire ou transformer leurs rapports, bref la configuration de leur société. L'étude de la parenté par les sciences sociales est plus avancée que l'étude des systèmes politiques, et ceux-ci sont peut-être plus facilement analysables que les systèmes religieux et les pratiques rituelles. Ceci peutêtre pour la bonne raison qu'au cœur des rapports sociaux existent des composantes imaginaires qui font partie de ces rapports et qui s'expriment et se vivent sous des formes symboliques. Or, la charge en composantes imaginaires et symboliques des systèmes religieux est certainement plus forte encore que celle contenue dans les systèmes politiques ou de parenté. 


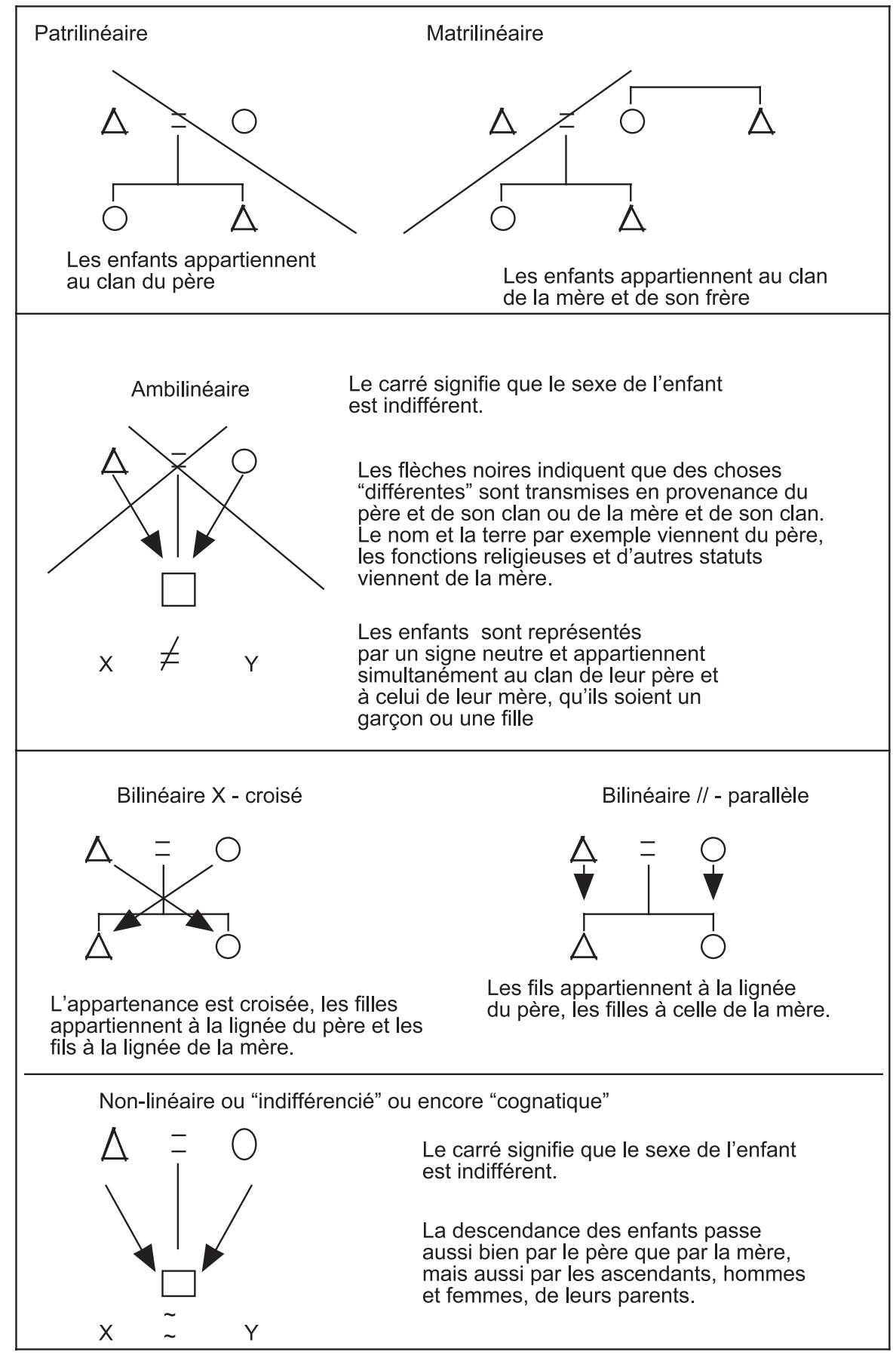

Fig. 3. Les différents principes de descendance.

On comprend donc la difficulté qu'il y a à analyser les faits sociaux compte tenu de la nature et de la complexité des éléments qui les composent, et de l'impossibilité d'expérimenter directement sur eux. Il devrait cependant être clair également que les sciences sociales n'ont pu progresser dans la connaissance des faits sociaux qu'en supposant que ces faits ont une logique, que les rapports sociaux tendent à faire système. Le système capitaliste de production et d'échanges marchands en est un exemple qui se développe sous nos yeux et dont nous sommes tous, à des degrés divers, à la fois les objets et les sujets. On comprendra aussi qu'il a donc fallu inventer des méthodes d'observation et d'analyse capables de saisir des fragments de ces logiques, globales ou locales, et qu'il faut traduire ces résultats dans des textes qui ne relèvent pas forcément de l'incantation littéraire, ou d'interprétations purement subjectives des faits observés. En bonne réciprocité, il faudrait maintenant que l'on nous parle des parties molles des sciences dures, de la sociobiologie par exemple? 